\title{
Synthesis and phase behavior of dendrons derived from 3,4,5-tris(tetradecyloxy)benzoic acid with different functional groups in focal point
}

\author{
MATVEY GRUZDEV ${ }^{\mathrm{a}, *}$, ULYANA CHERVONOVA $^{\mathrm{a}}$, OLGA AKOPOVA ${ }^{\mathrm{b}}$ \\ and ARKADIY KOLKER ${ }^{\mathrm{a}}$ \\ ${ }^{a}$ G A Krestov Institute of Solution Chemistry of the Russian Academy of Sciences, Ivanovo 153045, Russia \\ ${ }^{b}$ Nanomaterials Research Institute, Ivanovo State University, Ivanovo 153025, Russia \\ e-mail: gms@isc-ras.ru
}

MS received 18 February 2015; revised 12 May 2015; accepted 20 July 2015

\begin{abstract}
A number of dendrons of various chain lengths derived from the esters of 3,4,5-tris(tetradecyloxy)benzoic acid were synthesized. These esters were used as building blocks in the design of polyester molecules. Intermediate products, such as branched compounds with variation of functional groups in focal point (aromatic acids and their benzyl esters, aldehydes of different generation) were obtained. The structure and purity of all the compounds were determined by elemental analysis, FT-IR, NMR spectroscopy, mass spectrometry (MALDI-ToF). The phase behavior was investigated by differential scanning calorimetry and confirmed by polarized optical microscopy. Consequently, it was established that the liquid-crystalline properties of this series of dendrons arise from the degree of branching. This behavior can be explained by the formation of hydrogen bonds, as well as microsegregation processes of the links of the macromolecule.
\end{abstract}

Keywords. Dendron; building block; derivatives of benzoic acids; liquid crystalline properties; branching degree; microsegregation.

\section{Introduction}

Dendrimers and dendrons are highly branched globular macromolecules with precise structures, prepared through iterative synthesis. ${ }^{1}$ Current architectures that enable the elaboration of self-assembling dendrons that provide supramolecular dendrimers which are able to self-organize in periodic arrays ${ }^{2}$ are elaborated from dendrons containing anisotropic mesogenic repeat units, ${ }^{3-5}$ mesogenic dendrons and dendrimers, ${ }^{6}$ amorphous or liquid dendrimers containing mesogenic groups on their periphery and from polymer backbones dendronized with self-assembling dendrons. ${ }^{7,8}$ It is known that altering the end group functionality, ${ }^{9,10}$ as well as other structural modifications, ${ }^{11}$ influence the thermal properties of the dendritic molecules. The thermotropic liquid crystal mesophases of dendritic molecules containing 3,4-bis-dodecyloxybenzoic acid, 3,5-bis-dodecyloxybenzoic acid or 3,4,5-trisubstituted benzyl ether monodendrons moieties, in the periphery and as monodendrons, and other feasible monodendrons, have been studied extensively by Percec et al. ${ }^{10,12-14}$

*For correspondence
Commonly, branched oligomers can be described as calamitic molecules with disturbed linearity. One of the factors which promotes the development of mesomorphic properties, along with formation of dimers by hydrogen bonds, ${ }^{15}$ is microsegregation ${ }^{16}$ of the flexible nonpolar periphery and the rigid structure of the polar focal point of the dendron. Indeed, lateral substituents, such as $\mathrm{C}_{\mathrm{n}} \mathrm{H}_{2 \mathrm{n}+1}$, abruptly reduce the clearing point with increasing length, and influence the stability of mesophase by suppressing it existence. ${ }^{17}$ Previously we synthesized mesomorphic aldehydes comprizing a long alkyl residue, which acted as building blocks in the production of iron (III) metallocomplexes which showed spin-crossover properties..$^{18,19}$

The purpose of the present research is the synthesis of monodendrons with different degrees of branching, derived from 3,4,5-tris(tetradecyloxy)benzoic acid, and determination of the dependence of phase behavior on molecular structure. Thus prepared dendrons are used as precursor to Schiff bases in complexation reaction of spin-cross over metal containing systems. ${ }^{20}$ Recently, magnetic properties of a new dendrimeric spin crossover $\mathrm{Fe}(\mathrm{III})$ complex, $\left[\mathrm{Fe}(\mathrm{L})_{2}\right]^{+} \mathrm{PF}_{6}^{-}$, where $\mathrm{L}=$ 3,5-di[3,4,5-tris(tetradecyloxy)benzoyloxy]benzoyl-4salicylidene-N-ethyl-N-ethylenediamine, was reported 
for the first time. ${ }^{20}$ EPR studies show that this compound undergoes a gradual spin transition in the temperature range $70-300 \mathrm{~K}$ and has antiferromagnetic ordering below $10 \mathrm{~K}$. Mössbauer spectroscopy at $5 \mathrm{~K}$ confirms the presence of magnetic ordering in the dendrimeric iron complex.

\section{Experimental}

\subsection{Materials and Methods}

All the reagents and solvents were of chemically pure grade and were used without further purification. Benzyl ester of 3,5-dihydroxybenzoic acid was synthesized by the method in reference. ${ }^{21}$ FT-IR spectra of compounds were recorded on a Bruker Vertex $80 \mathrm{~V}$ device in the region of $7500-370 \mathrm{~cm}^{-1}$ in $\mathrm{KBr}$ pellets. The NMR spectral studies on the nuclei ${ }^{1} \mathrm{H}(500.17 \mathrm{MHz})$ and ${ }^{13} \mathrm{C}$ $(125.76 \mathrm{MHz})$ were recorded on a Bruker Avance-500 spectrometer. Elemental analysis of crystalline compounds was carried out on a FlashEA 1112 analyzer. The standard error of measurement is plus or minus $0.9 \%$. Mass-spectra were obtained using the MALDIToF method on a Bruker Daltonics Ultraflex massspectrometer in the positive ion-mode using reflection option; the matrix was 2,5-dihydroxybenzoic acid. Thin layer chromatography was performed on the chromatographic plates PolyGRAM, Sil G/UV 254; the eluent was chloroform. Thermopolarization microscopy was performed on a MIN-8 microscope with a heating plate of a custom design. Differential scanning calorimetry measurements were carried out on a NETZCH DSC 204 F1 device in aluminium capsules, the weight of the sample $\approx 10 \mathrm{mg}$, the heating rate was $10^{\circ} \mathrm{C} / \mathrm{min}$ in $\mathrm{N}_{2}$ atmosphere.

\subsection{Synthesis of the dendrons}

2.2a 3,4,5-Tris(tetradecyloxy)benzoic acid (1): The batches of tetradecyl bromide $(20.22 \mathrm{~g} ; 72.92 \mathrm{mmol})$ and ethyl ester of 3,4,5-trihydroxybenzoic acid $(4.89 \mathrm{~g}$; $28.74 \mathrm{mmol}$ ) were dissolved in $200 \mathrm{~mL}$ acetone. Then $4 \% \mathrm{NaOH}$ in $\mathrm{C}_{2} \mathrm{H}_{5} \mathrm{OH}$ was added, and the mixture was refluxed for $8 \mathrm{~h}$. The reaction was monitored by thinlayer chromatography (TLC). The reaction mixture was poured out into $1 \mathrm{M}$ solution of $\mathrm{HCl}$. After two days the solid residue was filtered off and the solvent was distilled in vacuum. The product was dissolved in diethyl ether and this solution was purified by chromatography on aluminum oxide. The solvent was removed on a rotary evaporator. Yield: $15.00 \mathrm{~g}(80 \%)$. MS MALDI-ToF $(\mathrm{m} / \mathrm{z})$ : found 781.94 , calculated 782.25
$\left(\mathrm{M}^{+}+\mathrm{Na}\right)$. $\mathrm{CHNO}$ analysis (\%): Calcd. for $\mathrm{C}_{49} \mathrm{H}_{90} \mathrm{O}_{5}$ : C 77.52; H 11.94; O 10.54. Found: C 76.78; H 12.59; O 10.63. ${ }^{1} \mathrm{H}$ NMR $\left(\mathrm{CDCl}_{3}\right.$, TMS, $\left.\delta, \mathrm{ppm}\right): 0.81$ (t, $9 \mathrm{H}$, $\mathrm{CH}_{3}$-Alk, J=7.32 Hz); 1.19 (m, 54H, $-\mathrm{CH}_{2}$-Alk); 1.41 (t, 6H, Alk- $\left.\mathrm{CH}_{2}-\mathrm{CH}_{2}-\mathrm{O}-, \mathrm{J}=6.71 \mathrm{~Hz}\right) ; 1.68(\mathrm{~m}, 6 \mathrm{H}$, $\mathrm{CH}_{2}$-Alk); 1.75 (m, 6H, - $\mathrm{CH}_{2}$-Alk); 3.95 (m, 6H, Alk$\mathrm{CH}_{2}-\mathrm{O}$ ); 7.19 (s, 2H, Ph-H); 11.98 (s, 1H, COOH). ${ }^{13} \mathrm{C}$ NMR $\left(\mathrm{CDCl}_{3}\right.$, TMS, $\left.\delta, \mathrm{ppm}\right): 14.11\left(\mathrm{CH}_{3}-\right), 22.69$ $\left(\mathrm{CH}_{3}-\mathrm{CH}_{2}-\right), 26.06\left(\mathrm{CH}_{3}-\mathrm{CH}_{2}-\underline{\mathrm{CH}}_{2}-\right), 29.70\left(\mathrm{CH}_{2}-\mathrm{Alk}\right)$, $31.92\left(\mathrm{O}-\mathrm{CH}_{2}-\mathrm{CH}_{2}-\mathrm{CH}_{2}-\right), 69.14\left(\mathrm{O}-\mathrm{CH}_{2}-\mathrm{Alk}\right), 73.52$ $\left(\mathrm{O}-\mathrm{CH}_{2}-\underline{\mathrm{CH}}_{2}-\right), 108.47\left(\mathrm{CH}_{\text {arom }}\right), 123.58\left(\mathrm{C}_{\text {arom }}\right), 143.10$ $\left(\mathrm{C}_{\text {arom }}\right), 152.82\left(\mathrm{C}_{\text {arom }}\right), 171.87(\mathrm{C}(\mathrm{O}) \mathrm{OH})$. FT-IR $(\mathrm{KBr})$ $v_{\max } / \mathrm{cm}^{-1}: 4326,4250$ (intermolecular hydrogen bond), $3418(\mathrm{OH}), 3081$ (aromatic vibrations of $\mathrm{C}-\mathrm{H}), 2921$ $2850\left(-\mathrm{CH}_{2}-\right), 2637-2530\left(-\mathrm{CH}_{3}-\right), 1688(\mathrm{C}=\mathrm{O}), 1130$ (-C-O-C-), 989-969 (flat deformational C-H vibrations of 1,3,4,5-substituted aromatic ring), $936(-\mathrm{OH}), 866$ (non-planar deformational $\mathrm{C}-\mathrm{H}$ vibrations of 1,3,4,5substituted aromatic ring).

2.2b 3,4,5-Tris(tetradecyloxy)benzoyloxy-2-hydroxybenzaldehyde (2): A weighed sample of compound (1) $(6.00 \mathrm{~g} ; 7.9 \mathrm{mmol})$ was dissolved in dichlormethane. 2,4-Dihydroxybenzaldehyde $(1.09 \mathrm{~g} ; 7.9 \mathrm{mmol})$ was added and the mixture was stirred until complete dissolution of the components. Then a sample of dicyclohexylcarbodiimide $(2.25 \mathrm{~g} ; 10.9 \mathrm{mmol})$ was added. The reaction mixture was stirred for $30 \mathrm{~min}$. After that, a catalytic amount of dimethylaminopyridine was added and the mixture was stirred for $12 \mathrm{~h}$. Precipitated urea was filtered off on a glass filter. The solvent was removed on a rotary evaporator. The residue was chromatographed on silica gel by chloroform. The product was freeze dried from benzene. Yield: $5.61 \mathrm{~g}$ (80.6\%). MS MALDI-ToF $(\mathrm{m} / \mathrm{z})$ : found 902.33 , calculated $902.36\left(\mathrm{M}^{+}+\mathrm{Na}\right)$. CHNO analysis (\%): Calcd. for $\mathrm{C}_{56} \mathrm{H}_{94} \mathrm{O}_{7}$ : C 76.49; $\mathrm{H} 10.77$; O 12.74. Found: $\mathrm{C}$ 75.93; H 11.17; O 12.70. ${ }^{1} \mathrm{H}$ NMR $\left(\mathrm{CDCl}_{3}\right.$, TMS, $\delta, \mathrm{ppm}): 0.81\left(\mathrm{t}, 9 \mathrm{H}, \mathrm{CH}_{3^{-}}, \mathrm{J}=6.10 \mathrm{~Hz}\right) ; 1.28-1.19$ (m, 54H, - $\left.\mathrm{CH}_{2}-\mathrm{Alk}\right) ; 1.41$ (t, 6H, Alk- $\mathrm{CH}_{2}-\mathrm{CH}_{2}-\mathrm{O}-$, $\mathrm{J}=6.10 \mathrm{~Hz}) ; 1.69\left(\mathrm{~m}, 6 \mathrm{H},-\mathrm{CH}_{2}-\mathrm{Alk}\right) ; 1.76(\mathrm{~m}, 6 \mathrm{H}$, $\left.-\mathrm{CH}_{2}-\mathrm{Alk}\right) ; 3.97$ (m, 6H, Alk- $\left.\mathrm{CH}_{2}-\mathrm{O}\right)$; 6.83-6.79 (d, $2 \mathrm{H}, \mathrm{Ph}-\mathrm{H}, \mathrm{J}=7.93 \mathrm{~Hz}$ ); 7.19 (s, $1 \mathrm{H}, \mathrm{Ph}-\mathrm{H}) ; 7.56-7.54$ (d, 2H, Ph-H, J=8.54 Hz); 9.82 (s, 1H, OH-); 11.19 (s, $1 \mathrm{H}, \mathrm{COH}) .{ }^{13} \mathrm{C} \mathrm{NMR}\left(\mathrm{CDCl}_{3}, \mathrm{TMS}, \delta, \mathrm{ppm}\right)$ : $14.15\left(\mathrm{CH}_{3}^{-}\right), 22.72\left(\mathrm{CH}_{3}-\underline{\mathrm{CH}}_{2}{ }^{-}\right), 26.09\left(-\mathrm{CH}_{2}-\right), 29.41$ $\left(-\mathrm{CH}_{2}-\right), 29.72\left(-\mathrm{CH}_{2}-\right), 29.74\left(-\mathrm{CH}_{2}-\right), 30.37\left(-\mathrm{CH}_{2}-\right)$, $31.96\left(\mathrm{CH}_{3}-\mathrm{CH}_{2}-\mathrm{CH}_{2}-\right), 69.30$ (-O-CH (-O-CH $2-\mathrm{Alk}), 108.64\left(\mathrm{CH}_{\text {arom }}\right), 110.98 \quad\left(\mathrm{CH}_{\text {arom }}\right)$, $114.18\left(\mathrm{CH}_{\text {arom }}\right), 118.69\left(\mathrm{C}_{\text {arom }}\right), 123.06\left(\mathrm{C}_{\text {arom }}\right), 134.97$ $\left(\mathrm{CH}_{\text {arom }}\right), 143.39\left(\mathrm{C}_{\text {arom }}\right), 153.04\left(\mathrm{C}_{\text {arom }}\right), 157.82\left(\mathrm{C}_{\text {arom }}\right)$, $163.21\left(\mathrm{C}_{\text {arom }}-\mathrm{OH}\right), 164.06(\mathrm{C}(\mathrm{O}) \mathrm{O}), 195.52(\mathrm{CHO})$. 
FT-IR (KBr) $v_{\max } / \mathrm{cm}^{-1}: 4329,4253$ (intermolecular hydrogen bond), $3433(\mathrm{OH}), 3091$ (aromatic $\mathrm{CH}$ vibrations), 2919-2848 $\left(\mathrm{CH}_{2}-\right), 1727(\mathrm{C}=\mathrm{O}), 1386-1274$ (Ph-CHO), 1193 (Alk-C-O-C(Ph)), 982 (flat deformational $\mathrm{C}$-H vibrations of 1,3,4,5-substituted aromatic ring), $873(\mathrm{OH})$.

2.2c Benzyl ester of 3,5-di[3,4,5-tris(tetradecyloxy) benzoyloxy]benzoic acid (3): A weighed sample of compound (1) (13.11 g; $17.27 \mathrm{mmol})$ was dissolved in dichlormethane. Benzyl ester of 3,5-dihydroxybenzoic acid $(2.11 \mathrm{~g} ; 8.64 \mathrm{mmol})$ was added and the mixture was stirred until complete dissolution of the components. Then a sample of dicyclohexylcarbodiimide (3.02 g; $14.63 \mathrm{mmol}$ ) was added and the reaction mixture was stirred for $30 \mathrm{~min}$. After that, a catalytic amount of dimethylaminopyridine was added and the mixture was stirred for $12 \mathrm{~h}$. Precipitated urea was filtered off on a glass filter. The solvent was removed on a rotary evaporator. The residue was chromatographed on silica gel by chloroform. The product was recrystallized from a 1:1 acetonitrile-chloroform mixture and filtered off. Yield: $13.21 \mathrm{~g}$ (88.53\%). MS MALDI-ToF $(\mathrm{m} / \mathrm{z})$ : found 1748.22 , calculated $1749.71\left(\mathrm{M}^{+}+\mathrm{Na}\right)$. CHNO analysis (\%): Calcd. for $\mathrm{C}_{112} \mathrm{H}_{188} \mathrm{O}_{12}$ : C 77.91; H 10.97; O 11.12. Found: C 77.41; H 11.84; O 10.75. ${ }^{1} \mathrm{H}$ NMR $\left(\mathrm{CDCl}_{3}\right.$, TMS $\left.\delta, \mathrm{ppm}\right): 0.89$ (t, $18 \mathrm{H}$, Alk$\left.\mathrm{CH}_{3}, \mathrm{~J}=5.49 \mathrm{~Hz}\right) ; 1.34-1.26\left(\mathrm{~m}, 108 \mathrm{H},-\mathrm{CH}_{2}-\mathrm{Alk}\right)$; 1.49 (s, $\left.12 \mathrm{H},-\mathrm{CH}_{2}-\mathrm{Alk}\right) ; 1.76$ (m, $\left.12 \mathrm{H},-\mathrm{O}-\mathrm{CH}_{2}-\mathrm{Alk}\right)$; $1.83\left(\mathrm{~m}, 12 \mathrm{H},-\mathrm{CH}_{2}-\mathrm{Alk}\right) ; 4.04$ (m, 12H, Alk- $\underline{\mathrm{CH}}_{2}-\mathrm{O}-$ ); 5.38 (s, 2H, O-CH $\left.{ }_{2}-\mathrm{Ph}\right) ; 7.32$ (s, 2H, Ph-H); 7.36 (s, 2H, Ph-H); 7.39 (m, 4H, Ph-H); 7.44 (s, 2H, Ph$\mathrm{H}) ; 7.83(\mathrm{~s}, 2 \mathrm{H}, \mathrm{Ph}-\mathrm{H}) .{ }^{13} \mathrm{C} \mathrm{NMR}\left(\mathrm{CDCl}_{3}, \mathrm{TMS}, \delta\right.$, ppm): $14.14\left(\mathrm{CH}_{3}-\right), 22.71\left(\mathrm{CH}_{3}-\mathrm{CH}_{2}-\right), 26.09$ (-O$\left.\mathrm{CH}_{2}-\mathrm{CH}_{2}-\mathrm{CH}_{2}-\right), 29.41\left(\mathrm{CH}_{3}-\mathrm{CH}_{2}-\mathrm{CH}_{2}-\mathrm{CH}_{2}-\right), 29.66$ (-O-CH$\left.{ }_{2}-\mathrm{CH}_{2}-\right), 30.37$ (- $\left.\mathrm{CH}_{2}-\mathrm{Alk}\right), 31.95\left(\mathrm{CH}_{3}-\mathrm{CH}_{2}-\right.$ $\left.\underline{\mathrm{CH}}_{2}-\right), 67.31 \quad\left(-\mathrm{CH}_{2}-\right), 69.27 \quad\left(-\mathrm{O}-\mathrm{CH}_{2}-\mathrm{Alk}\right), 73.61$ (-O-CH ${ }_{2}$-Alk), 108.57 ( $\left.\mathrm{CH}_{\text {arom }}\right), 120.64 \quad\left(\mathrm{CH}_{\text {arom }}\right)$, $123.16\left(\mathrm{CH}_{\text {arom }}\right), 128.45\left(\mathrm{CH}_{\text {arom }}\right), 128.67\left(\mathrm{CH}_{\text {arom }}\right)$, $132.34\left(\mathrm{CH}_{\text {arom }}\right), 135.56\left(\mathrm{C}_{\text {arom }}\right), 143.26\left(\mathrm{C}_{\text {arom }}\right), 151.42$ $\left(\mathrm{C}_{\text {arom }}\right), 153.02\left(\mathrm{C}_{\text {arom }}\right), 164.51(\mathrm{C}=\mathrm{O}), 164.84(\mathrm{C}=\mathrm{O})$. FT-IR (KBr), $v_{\max } / \mathrm{cm}^{-1}$ : 4321, 4253 (intermolecular hydrogen bond), $3414(\mathrm{OH}), 3069$ (aromatic vibrations of C-H), 2922-2849 (-CH $\left.2^{-}\right), 2631\left(-\mathrm{CH}_{3}-\right), 1729$ $(\mathrm{C}=\mathrm{O}), 1228-1194$ (flat deformational $\mathrm{C}-\mathrm{H}$ vibrations of 1,3,4,5-substituted aromatic ring), 1115 (-C-O-C-), 890-856 (non-planar deformational $\mathrm{C}-\mathrm{H}$ vibrations of 1,3,4,5-substituted aromatic ring).

2.2d 3,5-Di[3,4,5-tris(tetradecyloxy)benzoyloxy] benzoic acid (4): The calculated amount of catalyst 5\% $\mathrm{Pd} / \mathrm{C}(0.56 \mathrm{~g})$ was mixed with dioxane and activated with hydrogen in a steel hydrogenation reactor. After that, a solution of $8.46 \mathrm{~g}$ of compound (3) in $100 \mathrm{~mL}$ of dioxane was added and the reaction mixture was stirred at $50^{\circ} \mathrm{C}$ under hydrogen $(4 \mathrm{~atm})$ until the necessary amount of hydrogen was consumed. The reaction mixture was twice filtered through a glass filter, and solvent was removed on a rotary evaporator. The residue was dissolved in benzene and freeze dried to obtain a white solid product. Yield: $5.01 \mathrm{~g}(62.5 \%)$. MS MALDI-ToF $(\mathrm{m} / \mathrm{z})$ : found 1657.06 , calculated $1659.59\left(\mathrm{M}^{+}+\mathrm{Na}\right)$. CHNO analysis (\%): Calcd. for $\mathrm{C}_{105} \mathrm{H}_{182} \mathrm{O}_{12}$ : C 77.06; H 11.21; O 11.73. Found: C 76.65; H 12.10; O 11.25. ${ }^{1} \mathrm{H}$ NMR $\left(\mathrm{CDCl}_{3}, \mathrm{TMS}, \delta, \mathrm{ppm}\right): 0.80$ (m, 18H, Alk$\left.\mathrm{CH}_{3}\right) ; 1.18$ (m, 54H, - $\mathrm{CH}_{2}$-Alk); 1.42 (s, $12 \mathrm{H}, \mathrm{O}-\mathrm{CH}_{2}-$ $\mathrm{CH}_{2}$-Alk); 1.69 (m, 12H, - $\mathrm{CH}_{2}$-Alk); 1.77 (m, 12H, $-\mathrm{CH}_{2}$-Alk); 3.97 (m, 12H, O-CH $\mathrm{CH}_{2}$-Alk); 7.33 (s, 4H, Ph$\mathrm{H}) ; 7.35$ (s, 1H, Ph-H); 7.81 (d, 2H, Ph-H, J=1.83 Hz). ${ }^{13} \mathrm{C} \mathrm{NMR}\left(\mathrm{CDCl}_{3}\right.$, TMS, $\delta$, ppm): $14.15\left(\mathrm{CH}_{3}-\right), 22.71$ $\left(\mathrm{CH}_{3}-\mathrm{CH}_{2}-\right), 26.11$ (- $\left.\mathrm{CH}_{2}-\mathrm{Alk}\right), 29.41-29.77 \quad\left(-\mathrm{CH}_{2}-\right.$ $\mathrm{Alk}), 30.37$ (-O- $\left.\mathrm{CH}_{2}-\mathrm{CH}_{2}-\right), 31.96\left(\mathrm{CH}_{3}-\mathrm{CH}_{2}-\mathrm{CH}_{2}-\right)$, 69.29 (-O-CH $\left.{ }_{2}-\mathrm{Alk}\right), 73.63$ (-O-CH $\left.2-\mathrm{Alk}\right), 108.57$ $\left(\mathrm{CH}_{\text {arom }}\right), 123.48\left(\mathrm{C}_{\text {arom }}\right), 143.29\left(\mathrm{C}_{\text {arom }}\right), 152.97\left(\mathrm{C}_{\text {arom }}\right)$, $164.48(\mathrm{C}(\mathrm{O}) \mathrm{OH})$. FT-IR (KBr), $v_{\max } / \mathrm{cm}^{-1}: 4334,4249$ (intermolecular hydrogen bond), $3414(\mathrm{OH}), 3088$ (aromatic vibrations of $\mathrm{C}-\mathrm{H}), 2920-2852\left(-\mathrm{CH}_{2}-\right), 2632$ $\left(-\mathrm{CH}_{3}-\right), 1751(\mathrm{C}=\mathrm{O}), 1122$ (-C-O-C), 999 (flat deformational $\mathrm{C}$-H vibrations of 1,3,4,5-substituted aromatic ring), 924 (-OH), 856 (non-planar deformational C-H vibrations of 1,3,4,5-substituted aromatic ring).

2.2e 3,5-Di[3,4,5-tris(tetradecyloxy)benzoyloxy]benzoyl-4-oxy-2-hydroxybenzaldehyde (5): Compound (5) was obtained from (4) similar to (2) described earlier. The product was recrystallized from the 1:1 acetonitrile-chloroform mixture and filtered off. Yield: $1.37 \mathrm{~g}(63.63 \%)$. MS MALDI-ToF $(\mathrm{m} / \mathrm{z})$ : found 1778.19, calculated $1779.69\left(\mathrm{M}^{+}+\mathrm{Na}\right)$. CHNO analysis (\%): Calcd. for $\mathrm{C}_{112} \mathrm{H}_{186} \mathrm{O}_{14}$ : C 76.58; $\mathrm{H}$ 10.67; O 12.75. Found: C 76.48; H 11.15; O 12.37. ${ }^{1} \mathrm{H}$ NMR $\left(\mathrm{CDCl}_{3}, \mathrm{TMS}, \delta, \mathrm{ppm}\right): 0.81$ (t, $18 \mathrm{H}, \mathrm{CH}_{3}-\mathrm{Alk}, \mathrm{J}=6.71$ $\mathrm{Hz})$; $1.18-1.29$ (m, 108H, - $\mathrm{CH}_{2}$-Alk); 1.42 (m, 12H, $\left.-\mathrm{O}-\mathrm{CH}_{2}-\mathrm{CH}_{2}-\mathrm{Alk}\right) ; 1.69$ (m, 12H, - $\left.\mathrm{CH}_{2}-\mathrm{Alk}\right) ; 1.77$ (m, $\left.12 \mathrm{H},-\mathrm{CH}_{2}-\mathrm{Alk}\right) ; 3.98$ (m, $\left.12 \mathrm{H},-\mathrm{O}-\mathrm{CH}_{2}-\mathrm{Alk}\right) ; 6.79$ (s, 1H, Ph-H); 6.83 (m, 1H, Ph-H); 7.19 (s, 1H, Ph-H); 7.34 (m, 4H, Ph-H); 7.39 (s, 1H, Ph-H); 7.56 (s, 2H, $\mathrm{Ph}-\mathrm{H}) ; 7.89$ (m, 2H, Ph-H); 7.93 (s, 1H, Ph-H); 9.83 (s, $1 \mathrm{H}, \mathrm{OH}) ; 11.19$ (s, $1 \mathrm{H}, \mathrm{COH}) .{ }^{13} \mathrm{C} \mathrm{NMR}\left(\mathrm{CDCl}_{3}\right.$, TMS, $\delta$, ppm): $14.09\left(\mathrm{CH}_{3}{ }^{-}\right), 22.67\left(\mathrm{CH}_{3}-\mathrm{CH}_{2}-\right), 26.06$ (- $\left.\mathrm{CH}_{2}-\right), 29.26-30.33\left(-\mathrm{CH}_{2}-\right), 31.91\left(\mathrm{CH}_{3}-\mathrm{CH}_{2}-\mathrm{CH}_{2-}\right)$, 69.25 (-O- $\left.\mathrm{CH}_{2}-\mathrm{Alk}\right), 73.59$ (-O- $\left.\mathrm{CH}_{2}-\mathrm{Alk}\right), \quad 108.57$ $\left(\mathrm{CH}_{\text {arom }}\right), 114.01\left(\mathrm{CH}_{\text {arom }}\right), 120.92\left(\mathrm{C}_{\text {arom }}\right), 121.94$ $\left(\mathrm{CH}_{\text {arom }}\right), 122.91\left(\mathrm{CH}_{\text {arom }}\right), 130.72\left(\mathrm{C}_{\text {arom }}\right), 134.92$ 
$\left(\mathrm{CH}_{\text {arom }}\right), 143.37\left(\mathrm{C}_{\text {arom }}\right), 145.63\left(\mathrm{C}_{\text {arom }}\right), 151.65\left(\mathrm{C}_{\text {arom }}\right)$, $152.99\left(\mathrm{C}_{\text {arom }}\right), 163.17\left(\mathrm{C}_{\text {arom }}-\mathrm{OH}\right), 164.46(\mathrm{C}(\mathrm{O}) \mathrm{O})$, 195.48 (CHO). FT-IR (KBr), $v_{\max } / \mathrm{cm}^{-1}: 4326,4253$ (intermolecular hydrogen bond), $3420(-\mathrm{OH}), 3103$ (aromatic vibrations of $\mathrm{CH}), 2910-2850 \quad\left(-\mathrm{CH}_{2}-\right)$, 1738 (C=O), 1393-1288 (Ph-CHO), 1196 (Alk-C$\mathrm{O}-\mathrm{C}(\mathrm{Ph})$ ), 984 (flat deformational $\mathrm{C}-\mathrm{H}$ vibrations of 1,3,4,5-substituted aromatic ring), $873(\mathrm{OH})$.

2.2f Benzyl ester 3,5-di[3,5-bis(3,4,5-tris(tetradecyloxy) benzoyloxy)benzoyloxy]-benzoic acid (6): Compound (6) was obtained from (4) similar to (3). Yield: $6.38 \mathrm{~g}(70.58 \%)$. MS MALDI-ToF $(\mathrm{m} / z)$ : found 3494.08/3509.17, calculated $3499.39\left(\mathrm{M}^{+}+\mathrm{NH}_{4}\right) /$ $3504.39\left(\mathrm{M}^{+}+\mathrm{Na}\right)$. CHNO analysis (\%): Calcd. for $\mathrm{C}_{224} \mathrm{H}_{372} \mathrm{O}_{26}$ : C 77.28; H 10.77; O 11.95. Found: $\mathrm{C}$ 76.77; H 11.29; O 11.94. ${ }^{1} \mathrm{H}$ NMR $\left(\mathrm{CDCl}_{3}\right.$, TMS, $\delta$, ppm): 0.80 (m, 36H, $\mathrm{CH}_{3}$-Alk); 1.18-1.29 (m, 216H, $-\mathrm{CH}_{2}$-Alk); 1.42 (m, 24H, -O- $\left.\mathrm{CH}_{2}-\mathrm{CH}_{2}-\mathrm{Alk}\right) ; 1.69$ (m, $\left.24 \mathrm{H},-\mathrm{CH}_{2}-\mathrm{Alk}\right) ; 1.77$ (m, 24H, $\left.-\mathrm{CH}_{2}-\mathrm{Alk}\right) ; 3.98$ (m, 24H, -O- $\mathrm{CH}_{2}-\mathrm{Alk}$ ); 5.31 (s, 2H, -O- $\left.\mathrm{CH}_{2}-\mathrm{Ph}\right) ; 7.19$ (s, $1 \mathrm{H}, \mathrm{Ph}-\mathrm{H}) ; 7.27-7.29$ (d, 2H, Ph-H, J=7.32 Hz); 7.34 (d, 8H, Ph-H, J=14.04 Hz); 7.37-7.39 (d, 4H, Ph-H, $\mathrm{J}=8.54 \mathrm{~Hz}) ; 7.79$ (s, 2H, Ph-H); 7.90 (s, 3H, Ph-H). ${ }^{13} \mathrm{C} \mathrm{NMR}\left(\mathrm{CDCl}_{3}, \mathrm{TMS}, \delta, \mathrm{ppm}\right): 14.11\left(\mathrm{CH}_{3}-\right), 22.68$ $\left(\mathrm{CH}_{3}-\mathrm{CH}_{2-}\right), 26.07 \quad\left(-\mathrm{CH}_{2}-\right), 29.37\left(-\mathrm{CH}_{2}-\right), 29.69$ $\left(-\mathrm{CH}_{2}-\right), 30.33\left(-\mathrm{CH}_{2}-\right), 31.91\left(\mathrm{CH}_{3}-\mathrm{CH}_{2}-\mathrm{CH}_{2}-\right), 67.36$ (- $\left.\mathrm{CH}_{2}-\mathrm{Ph}\right), 69.24$ (-O-CH $\left.2-\mathrm{Alk}\right), 73.58$ (-O-C $\left.\mathrm{CH}_{2}-\mathrm{Alk}\right)$, $108.53\left(\mathrm{CH}_{\text {arom }}\right), 121.14\left(\mathrm{CH}_{\text {arom }}\right), 122.96\left(\mathrm{CH}_{\text {arom }}\right)$, $128.41\left(\mathrm{CH}_{\text {arom }}\right), 128.65\left(\mathrm{CH}_{\text {arom }}\right), 130.92\left(\mathrm{C}_{\text {arom }}\right)$, $143.28\left(\mathrm{C}_{\text {arom }}\right), 150.99\left(\mathrm{C}_{\text {arom }}\right), 151.61\left(\mathrm{C}_{\text {arom }}\right), 162.99$ $(\mathrm{C}(\mathrm{O}) \mathrm{O}), 164.44(\mathrm{C}(\mathrm{O}) \mathrm{O})$. FT-IR $(\mathrm{KBr}), v_{\max } / \mathrm{cm}^{-1}$ : 4335, 4253 (intermolecular hydrogen bond), 3420 (OH), 3094 (aromatic vibrations of C-H), 2929-2850 $\left(-\mathrm{CH}_{2}-\right), 2666\left(\mathrm{CH}_{3}-\right), 1738(\mathrm{C}=\mathrm{O}), 1187$ (flat deformational C-H vibrations of 1,3,4,5-substituted aromatic ring), 1127 (-C-O-C-), 892 (non-planar deformational $\mathrm{C}-\mathrm{H}$ vibrations of 1,3,4,5-substituted aromatic ring).

$2.2 \mathrm{~g}$ 3,5-Di[3,5-bis(3,4,5-tris(tetradecyloxy)benzoyloxy)benzoyloxy]-benzoic acid (7): Compound (7) was obtained from (6) similar to (4). Yield: $3.81 \mathrm{~g}$ $(70.41 \%)$. MS MALDI-ToF $(m / z)$ : found 3406.23, calculated $3409.30\left(\mathrm{M}^{+}+\mathrm{NH}_{4}\right)$. CHNO analysis $(\%)$ : Calcd. for $\mathrm{C}_{217} \mathrm{H}_{366} \mathrm{O}_{26}$ : C 76.86; H 10.88; O 12.27. Found: C 76.27; $\mathrm{H} 10.99$; O 12.74. ${ }^{1} \mathrm{H}$ NMR $\left(\mathrm{CDCl}_{3}\right.$, TMS, $\delta$, ppm): 0.80 (m, 36H, $\mathrm{CH}_{3}$-Alk); 1.18-1.29 (m, $\left.216 \mathrm{H},-\mathrm{CH}_{2}-\mathrm{Alk}\right) ; 1.42$ (m, 24H, -O- $\mathrm{CH}_{2}-\mathrm{CH}_{2}-\mathrm{Alk}$ ); 1.69 (m, 24H, - $\mathrm{CH}_{2}$-Alk); 1.76 (m, 24H, - $\mathrm{CH}_{2}-\mathrm{Alk}$ ); 3.98 (m, 24H, -O-CH - -Alk); 7.19 (s, 4H, Ph-H); 7.34 (s, 8H, Ph-H); 7.39 (s, 2H, Ph-H); 7.82 (s, 1H, Ph-H); 7.91 (s, 2H, Ph-H). ${ }^{13} \mathrm{C}$ NMR $\left(\mathrm{CDCl}_{3}\right.$, TMS, $\left.\delta, \mathrm{ppm}\right)$ :
$14.39\left(\mathrm{CH}_{3}{ }^{-}\right), 22.66\left(\mathrm{CH}_{3}-\mathrm{CH}_{2}-\right), 26.02\left(-\mathrm{CH}_{2}-\right), 26.07$ $\left(-\mathrm{CH}_{2}-\right), 29.28\left(-\mathrm{CH}_{2}-\right), 29.35\left(-\mathrm{CH}_{2}-\right), 30.31\left(-\mathrm{CH}_{2}^{-}\right)$, $31.89\left(\mathrm{CH}_{3}-\mathrm{CH}_{2}-\mathrm{CH}_{2}-\right), 69.24$ (-O-CH $\left.2-\mathrm{Alk}\right), 73.58$ (-O- $\mathrm{CH}_{2}$-Alk), $108.55\left(\mathrm{CH}_{\text {arom }}\right), 120.88 \quad\left(\mathrm{CH}_{\text {arom }}\right)$, $120.94\left(\mathrm{CH}_{\text {arom }}\right), 122.97\left(\mathrm{CH}_{\text {arom }}\right), 130.74\left(\mathrm{C}_{\text {arom }}\right)$, $131.19\left(\mathrm{C}_{\text {arom }}\right), 143.29\left(\mathrm{C}_{\text {arom }}\right), 149.48\left(\mathrm{C}_{\text {arom }}\right), 151.61$ $\left(\mathrm{C}_{\text {arom }}\right), \quad 152.99 \quad\left(\mathrm{C}_{\text {arom }}\right), \quad 164.44(\mathrm{C}(\mathrm{O}) \mathrm{O}), 167.14$ $(\mathrm{COOH})$. FT-IR $(\mathrm{KBr}), v_{\max } / \mathrm{cm}^{-1}$ : 4326, 4266 (intermolecular hydrogen bond), 3328 (OH), 3094 (aromatic vibrations of $\mathrm{CH}-), 2929-2850\left(-\mathrm{CH}_{2}-\right), 1738$ $(\mathrm{C}=\mathrm{O}), 1117$ (-C-O-C-), 1003 (flat deformational C-H vibrations of 1,3,4,5-substituted aromatic ring), 892 $(\mathrm{OH})$.

2.2h 3,5-Di[3,5-bis(3,4,5-tris(tetradecyloxy)benzoyloxy)benzoyloxy]-benzoyloxy-benzaldehyde (8): Compound (8) was obtained from (7) similar to (2). Yield: $2.51 \mathrm{~g}(68.39 \%)$. MS MALDI-ToF $(\mathrm{m} / \mathrm{z})$ : found 3519.97, calculated $3511.37\left(\mathrm{M}^{+}+\mathrm{NH}_{4}\right)$. CHNO analysis (\%): Calcd. for $\mathrm{C}_{224} \mathrm{H}_{370} \mathrm{O}_{28}$ : C 76.62; H 10.62; $\mathrm{O}$ 12.76. Found: C 77.13; H 10.63; O 12.24. ${ }^{1} \mathrm{H}$ NMR $\left(\mathrm{CDCl}_{3}\right.$, TMS, $\left.\delta, \mathrm{ppm}\right): 0.81\left(\mathrm{t}, 36 \mathrm{H}, \mathrm{CH}_{3}-\mathrm{Alk}\right.$, $\mathrm{J}=3.05 \mathrm{~Hz}) ; 1.19-1.29\left(\mathrm{~m}, 216 \mathrm{H},-\mathrm{CH}_{2}-\mathrm{Alk}\right) ; 1.42$ (m, $24 \mathrm{H}$, -O- $\left.\mathrm{CH}_{2}-\mathrm{CH}_{2}-\mathrm{Alk}\right) ; 1.69$ (m, 24H, - $\left.\mathrm{CH}_{2}-\mathrm{Alk}\right)$; 1.76 (m, 24H, - $\mathrm{CH}_{2}$-Alk); 3.98 (m, 24H, -O-CH $\left.2-A l k\right)$; 7.19 (s, 2H, Ph-H); 7.30 (s, 4H, Ph-H); 7.34 (s, 8H, Ph-H); 7.40 (m, 2H, Ph-H); 7.48 (s, 1H, Ph-H); 7.89 (s, 1H, Ph-H); 7.92 (s, 2H, Ph-H); 9.83 (s, 1H, OH); 11.19 (s, $1 \mathrm{H}, \mathrm{COH}) .{ }^{13} \mathrm{C} \mathrm{NMR}\left(\mathrm{CDCl}_{3}, \mathrm{TMS}, \delta, \mathrm{ppm}\right): 14.09$ $\left(\mathrm{CH}_{3}-\right), 22.68\left(\mathrm{CH}_{3}-\mathrm{CH}_{2-}\right), 26.07\left(-\mathrm{CH}_{2}-\right), 29.35-30.68$ $\left(-\mathrm{CH}_{2}-\right), 31.91\left(\mathrm{CH}_{3}-\mathrm{CH}_{2}-\mathrm{CH}_{2}-\right), 69.29\left(-\mathrm{O}-\mathrm{CH}_{2}-\mathrm{Alk}\right)$, 73.59 (-O-CH$-\mathrm{Alk}), 108.61\left(\mathrm{CH}_{\text {arom }}\right), 117.67\left(\mathrm{CH}_{\text {arom }}\right)$, $121.13\left(\mathrm{C}_{\text {arom }}\right), 122.98\left(\mathrm{C}_{\text {arom }}\right), 143.36\left(\mathrm{C}_{\text {arom }}\right), 151.63$ $\left(\mathrm{C}_{\text {arom }}\right), 153.03\left(\mathrm{C}_{\text {arom }}\right), 164.44(\mathrm{C}(\mathrm{O}) \mathrm{O}), 199.33(\mathrm{CHO})$. FT-IR (KBr), $v_{\max } / \mathrm{cm}^{-1}$ : 4326, 4253 (intermolecular hydrogen bond), $3315(\mathrm{OH}), 3103$ (aromatic vibrations of $\mathrm{CH}-), 2951-2859\left(-\mathrm{CH}_{2}-\right), 1738(\mathrm{C}=\mathrm{O}), 1393-1279$ (Ph-CHO), 1187 (Alk-C-O-C(Ph)), 1003 (flat deformational $\mathrm{C}$-H vibrations of 1,3,4,5-substituted aromatic ring), $883(\mathrm{OH})$.

\section{Results and Discussion}

3,4,5-Tris(tetradecyloxy)benzoic acid (1) was selected as the initial compound, and was synthesized in compliance with a standard method. ${ }^{22}$ For the regular branching, the method of doubling molecular size was employed, using the benzyl ester of 3,5-dihydroxybenzoic acid ${ }^{23,24}$ (scheme 1). The benzyl ester of 3,5-dihydroxybenzoic acid, employed as a protecting block in the esterification, was prepared by means of alkylation of the carboxyl group of 3,5-dihydroxybenzoic acid with 


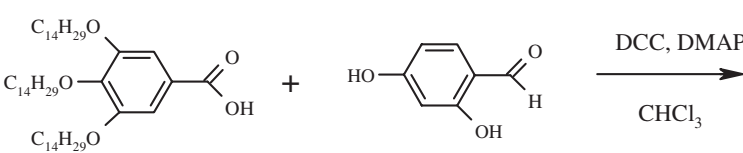

(1)

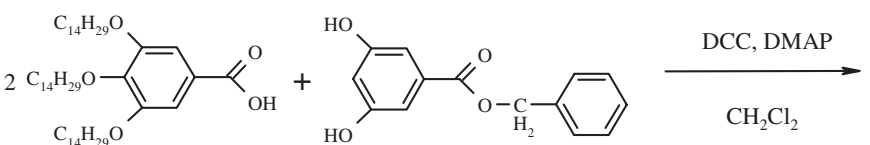

(1)
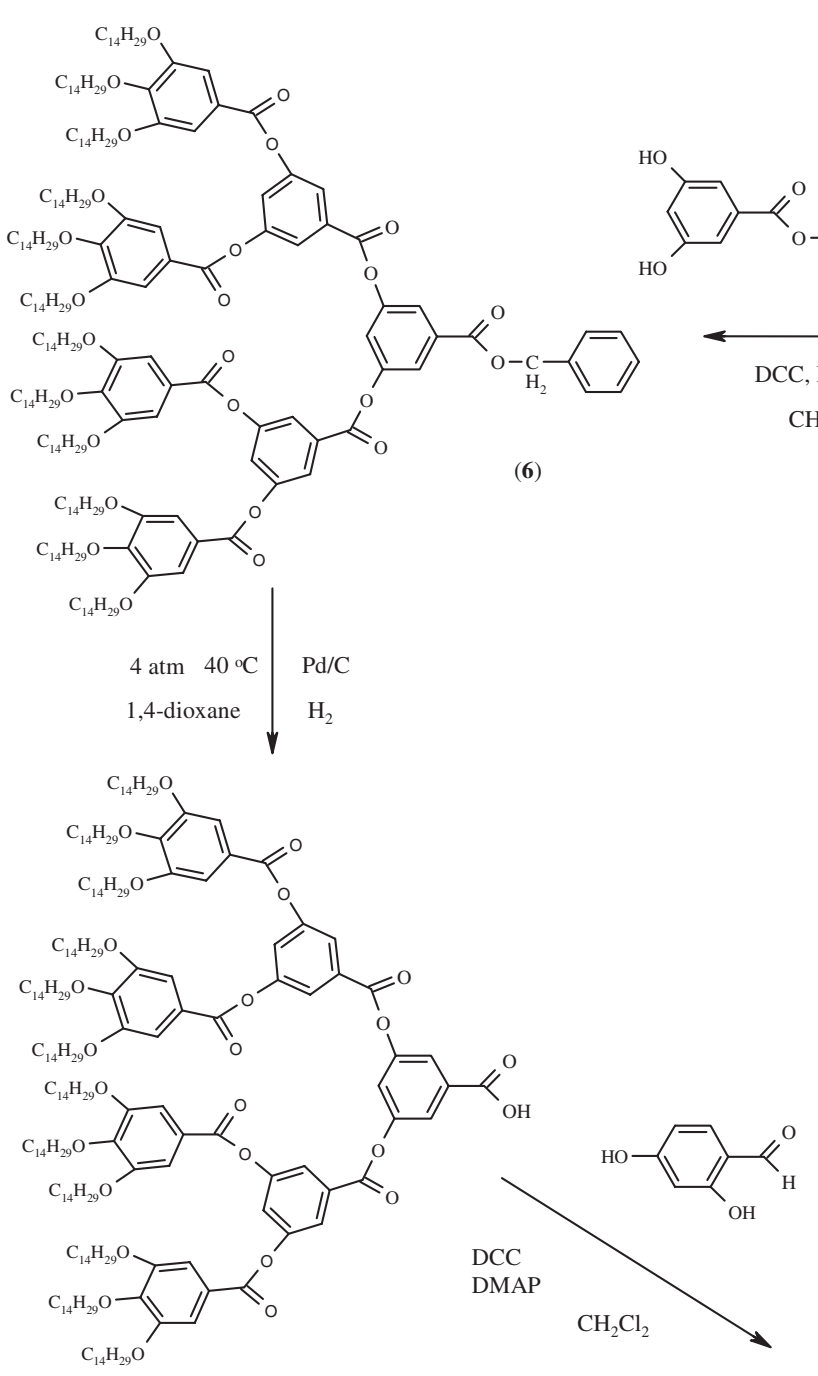

(7)

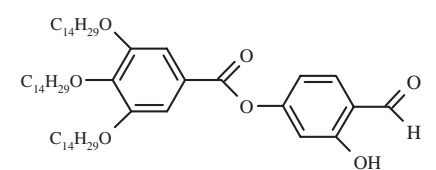

(2)

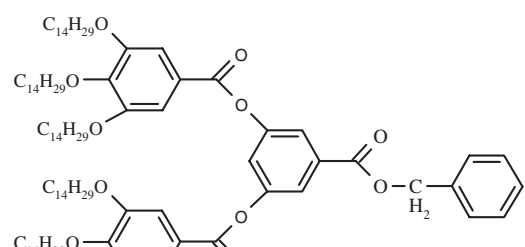

(3)
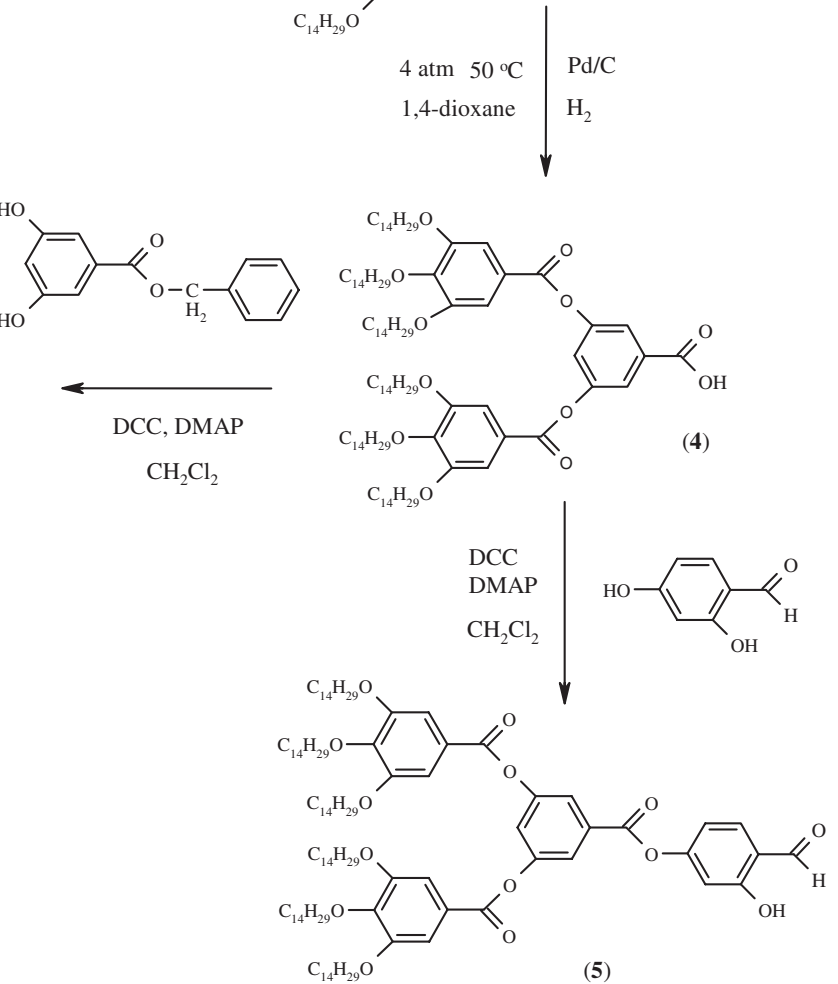

(5)

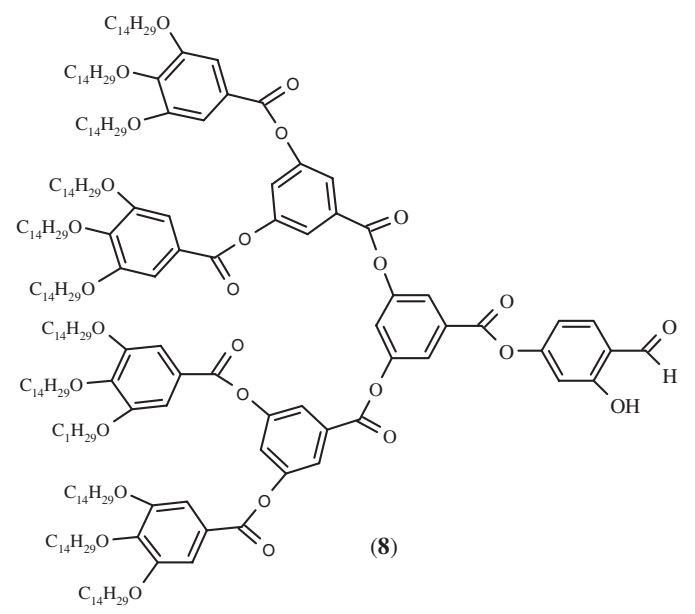

Scheme 1. Synthesis of compounds (1)-(8). 
benzyl bromide in dry DMF. ${ }^{25}$ The hydroxyl groups location in the structure of the benzyl ester of 3,5dihydroxybenzoic acid are sterically favorable for further molecular growth. The protecting group is easily introduced into the molecule, and selectively removed without any alterations in the structure of the formed compound. Additionally, the protecting group is stable in the presence of a large number of reagents used for further reactions.

The esterification was carried out by interaction between the carboxyl and hydroxyl groups with the aid of dicyclohexylcarbodiimide in dichloromethane. The benzyl group was selectively removed by hydrogenolysis using 5\% Pd-C as catalyst in dry 1,4-dioxane ${ }^{26}$ (scheme 1). The aldehydes (2), (5) and (8) were obtained by means of esterification of the corresponding acid and para-hydroxysalicylic aldehyde.

The structure of the compounds was established by FT-IR, NMR, MALDI-ToF mass-spectrometry (matrixassisted laser desorption/ionization) and elemental analysis. To aid identification of the products, reference markers were used. This allowed us to determine the structure and the extent of the reactions.

The results of NMR spectroscopy showed that the integrated intensity of the alkyl fragments of acids (1), (4) and (7) increases with generation number. The R$\mathrm{CH}_{2}-\mathrm{O}$ - fragments in the 3,4,5-position of gallic acid are used as reference signals which characterize the molecular growth, i.e. the ratio of protons in $\mathrm{R}-\mathrm{CH}_{2}-$ $\mathrm{O}-$ to acids $(\mathbf{1}) /(\mathbf{4}) /(\mathbf{7})$ is $6 / 12 / 24$, respectively.

For acids (1), (4) and (7), it was found that the molecular ions are stabilized by sodium and ammonium ions. The data of MALDI-ToF analyses are given in figure 1 . The mass of the molecular ions increased exponentially.

For esters (3) and (6), the formation of product after introducing the benzyl fragment was confirmed by the

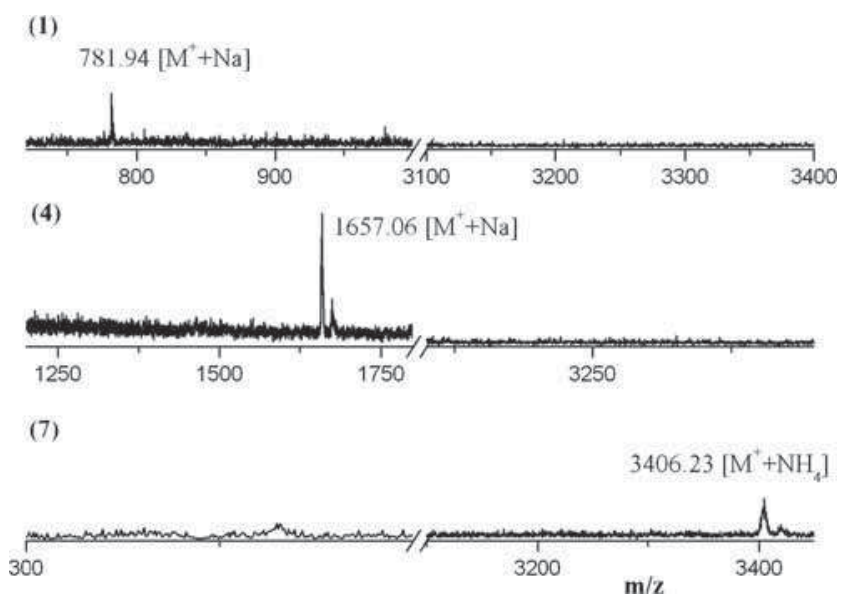

Figure 1. MS MALDI-ToF spectra of acids (1), (4), and (7). signal of $-\mathrm{CH}_{2}-\mathrm{Ph}$ at 5.38 and $5.31 \mathrm{ppm}$, respectively. Mass-spectra of compounds (3) and (6) demonstrate doubling of molecular mass from $1748\left[\mathrm{M}^{+}+\mathrm{Na}\right](3)$ to $3498\left[\mathrm{M}^{+}+\mathrm{NH}_{4}\right](\mathbf{6})$.

In the NMR spectra of compounds (2), (5), and (8), the signal of the $-\mathrm{COH}$ proton is located in the weak field range at $9.8 \mathrm{ppm}$, and the signal at $11.19 \mathrm{ppm}$ refers to the $-\mathrm{OH}$ proton. The mass-spectra of the aldehydes show molecular ions which are stabilized by sodium and ammonium ions. The values are doubled in series from (2) to (5) and from (5) to (8).

The thermal stability and phase behavior of compounds (1)-(8) were studied by differential scanning calorimetry and polarized optical microscopy. Consequently, it was established that liquid crystalline properties are detected with increasing branching degree in a homologous series, summarized in table 1 .

Thus, 3,4,5-tris(tetradecyloxy)benzoic acid (1) turns into an isotropic liquid at $\mathrm{T}_{\text {iso }}=70-71{ }^{\circ} \mathrm{C}$ in the heating cycle without forming a mesophase. Cooling of the sample initially leads to the growth of needle-shaped crystals. The data of DSC experiments confirm the results of polarized microscopy, table 1. Lengthening of compound (1) to (2) does not promote the formation of a mesophase. For this sample, a "solid-solid" type phase transition at $\mathrm{T}=31.96^{\circ} \mathrm{C}(\Delta \mathrm{H}=10.02 \mathrm{~kJ} / \mathrm{mol})$ and formation of an isotropic liquid at $\mathrm{T}_{\text {iso }}=64-65^{\circ} \mathrm{C}$ are characterized in the heating cycle. Cooling of the sample is accompanied by the growth of crystals, starting from $\mathrm{T}_{\mathrm{cr}}=34^{\circ} \mathrm{C}$. Data of DSC experiments confirm the results of microscopy.

In comparison with samples (1) and (2), compound (3) has a more branched structure and does not have terminal polar groups, which leads to the occurrence of enantiotropic mesomorphism (figure 2). After examination of sample (3) by polarized microscopy, it was established that a mesophase appears after reheating, i.e. upon second heating (figure 2c). Sample (3) flows anisotropically by pressing at $\mathrm{T}=30-48^{\circ} \mathrm{C}$. Upon cooling, the sample becomes too cold, and anisotropy appears at $\mathrm{T}=17^{\circ} \mathrm{C}$ as a nongeometric (planar) texture (figure 2a). Data of POM agrees with the differential scanning calorimetry data (figure 2b). As stated above, the feature in these kinds of esters is a protected active functional group which promotes the formation of a mesophase at the expense of microsegregation of the molecules. Upon first heating, the mesophase is difficult to detect because the system is very viscous and the compound is similar to an oligomer. After first heating and cooling, the sample is ordered, and this leads to the occurrence of a mesophase upon reheating (second heating). The exothermic peak in the heating process just before the peak at $\mathrm{T}=48.48^{\circ} \mathrm{C}$ corresponds to 
Table 1. The phase transition temperatures of compounds 1-8 according to DSC, during heating and cooling cycles.

\begin{tabular}{|c|c|c|}
\hline Compound & Thermal cycle & $\begin{array}{l}\text { Peak phase-transition temperatures }\left[{ }^{\circ} \mathrm{C}\right] \\
\quad(\text { transition enthalpies }[\mathrm{kJ} / \mathrm{mol}])\end{array}$ \\
\hline \multirow[t]{2}{*}{$\mathrm{C}_{49} \mathrm{H}_{90} \mathrm{O}_{5}(\mathbf{1})$} & $1^{\text {st }}$ heating & $\mathrm{Cr} 72.69(84.43) \rightarrow$ Iso \\
\hline & $1^{\text {st }}$ cooling & Iso $47.11(-84.51) \rightarrow \mathrm{Cr}$ \\
\hline \multirow[t]{2}{*}{$\mathrm{C}_{56} \mathrm{H}_{99} \mathrm{O}_{7}(\mathbf{2})$} & $1^{\text {st }}$ heating & Cr 31.96* $(10.02)^{*} \rightarrow \operatorname{Cr} 64.51(99.02) \rightarrow$ Iso \\
\hline & $1^{\text {st }}$ cooling & Iso $34.11(-80.11) \rightarrow \mathrm{Cr}$ \\
\hline \multirow[t]{2}{*}{$\mathrm{C}_{112} \mathrm{H}_{188} \mathrm{O}_{12}(\mathbf{3})$} & $2^{\text {nd }}$ heating & Cr $30.69(72.18) \rightarrow$ Mes $48.48(12.61) \rightarrow$ Iso \\
\hline & $2^{\text {nd }}$ cooling & Iso $17.61(-88.99) \rightarrow$ Mes $1.67(-1.21) \rightarrow \mathrm{Cr}$ \\
\hline \multirow[t]{2}{*}{$\mathrm{C}_{105} \mathrm{H}_{182} \mathrm{O}_{12}(\mathbf{4})$} & $1^{\text {st }}$ heating & $\mathrm{Cr} 47.61(33.88) \rightarrow$ Mes $50.68(-21.49) \rightarrow \operatorname{Cr} 82.34(102.45) \rightarrow$ Iso \\
\hline & $1^{\text {st }}$ cooling & Iso $30.89(-67.75) \rightarrow$ Mes $-66.14(-2.41)^{[\mathrm{a}]} \rightarrow \mathrm{Cr}^{[\mathrm{a}]}$ \\
\hline \multirow[t]{2}{*}{$\mathrm{C}_{112} \mathrm{H}_{182} \mathrm{O}_{14}(\mathbf{5})$} & $2^{\text {nd }}$ heating & Cr $31.96(26.53) \rightarrow$ Mes $55.49(20.03) \rightarrow$ Iso \\
\hline & $2^{\text {nd }}$ cooling & Iso $40.44(-8.43) \rightarrow$ Mes $20.34(-25.47) \rightarrow \mathrm{Cr}$ \\
\hline \multirow[t]{2}{*}{$\mathrm{C}_{224} \mathrm{H}_{370} \mathrm{O}_{28}(\mathbf{6})$} & $2^{\text {nd }}$ heating & Cr $49.51(218.28)^{*} \rightarrow$ Mes $59.98(*) \rightarrow$ Iso \\
\hline & $2^{\text {nd }}$ cooling & Iso $34.32(-107.82) \rightarrow$ Mes $^{[a]}$ \\
\hline \multirow{2}{*}{$\mathrm{C}_{217} \mathrm{H}_{366} \mathrm{O}_{26}(7)$} & $1^{\text {st }}$ heating & Cr $60.37(4.07) \rightarrow$ Iso \\
\hline & $1^{\text {st }}$ cooling & Iso $55.35(-24.76) \rightarrow$ Mes $14.29(-51.55)^{[b]}$ \\
\hline \multirow{2}{*}{$\mathrm{C}_{224} \mathrm{H}_{370} \mathrm{O}_{28}(\mathbf{8})$} & $1^{\text {st }}$ heating & $\begin{array}{c}\text { Cr } 50.01(11.24) \rightarrow \\
\rightarrow\end{array}$ \\
\hline & 1 cooming & 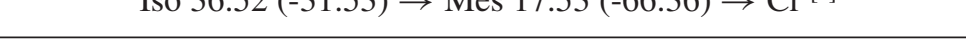 \\
\hline
\end{tabular}

Note: * - total area under curve; $\mathrm{Cr}$ - crystalline phase; Mes - mesophase; Iso - isotropic liquid. [a] Transition only observed after several day at room temperature. [b] Isotropic liquid at room temperature. No DSC peaks were detected below room temperature

some restructuring process in the development of the mesophase under heating.

Removal of the protective benzene group from compound (3) leads to an increase in the mesophase thermo stability of compound (4), and an extention of the temperature region of its existence (figure 3 ). Microscopic observations of sample (4) show that the mesophase with an uncharacteristic texture arises at $\mathrm{T}_{\text {mes }}=50^{\circ} \mathrm{C}$ during the heating cycle (texture is nonrelevant), and it turns into an isotropic liquid at $\mathrm{T}_{\text {iso }}=80^{\circ} \mathrm{C}$ via a mesophase transition (table 1). This process of crystallization is possible explained by aggregation of (4) which reaches a plateau after $\mathrm{T}_{\mathrm{cr}}=50^{\circ} \mathrm{C}$ within the investigated range of $49-71^{\circ} \mathrm{C} .{ }^{27}$ These aggregates are stable until further warming to $\mathrm{T}_{\text {iso }}$. Upon cooling, the sample becomes too cold and anisotropic parts appear at room temperature only after a long time of exposure of the sample. Figure 3 a shows an acinous fine domain texture, and against the background are large domains of mesophase which appear as a helical track. In addition, two phase transitions during the heating cycle are registered on the DSC curve (figure $3 b$ ). The thermograms of compounds (3) and (4) (by themselves) are not indicative of a mesophase, but the description of microscopic observation gives evidence for enantiotropic mesomorphism. Unfortunately, characteristic textures are not observed upon heating, but a mesophase is revealed by the fluidity of the compound upon pressure and a change of the rotation of crossed polarizers at 45 and 90 degrees. Undoubtedly, investigation of the compounds by X-ray diffraction might to help us to confirm mesomorphism, but under the condition that samples must be orientated. Unfortunately, such investigations are impossible owing to high viscosity. When we obtained the microphotos, we encountered complications owing to the considerable viscosity of the samples and difficulty in obtaining samples with a large domain texture.

Extention of compound (4) on the phenyl ring, and the presence of two polar groups in the ortho-position leads to a small decrease in the mesophase thermal stability (compound (5)). Characteristic texture mesophase of aldehyde (5) was not observed under first heating. A mesophase was observed only upon reheating. Anisotropy appears in the sample after 20-30 min. following cooling cycle.

Further modification of compound (4) to compound (6) leads to a reduction of the mesophase thermal stability, as compared with (4) (figure 4). Transition to an isotropic liquid is observed at $\mathrm{T}_{\text {iso }}=59.98^{\circ} \mathrm{C}$ (figure $4 \mathrm{c}$ ). The texture of a mesophase is more characteristic upon reheating. During cooling, anisotropy appears as a nongeometric texture, figure $4 \mathrm{a}$, for the thick sample, and as oily striations to the thin sample, figure $4 \mathrm{~b}$. Such behavior can denote a chirality of the mesophase.

Removal of the protective group from compound (6) impairs the conditions for development of mesomorphism in compound (7), which turns into an isotropic liquid upon melting $\left(\mathrm{T}_{\text {iso }}=60^{\circ} \mathrm{C}\right)$. During cooling, a texture with a great number of anisotropic points which lengthen, move, and change simultaneously were observed. The sample shows monotropic mesomorphism. 
(a)

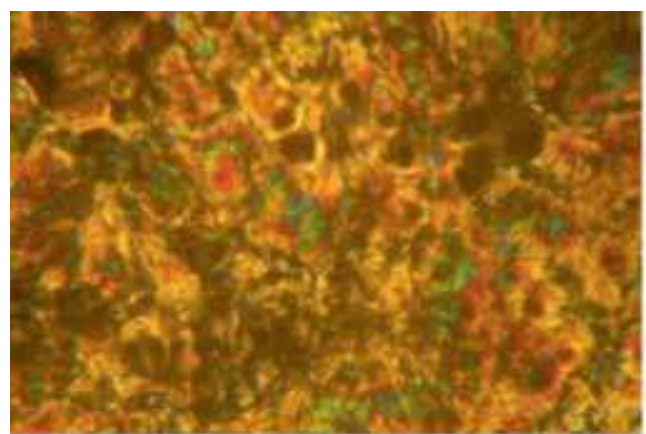

(b)

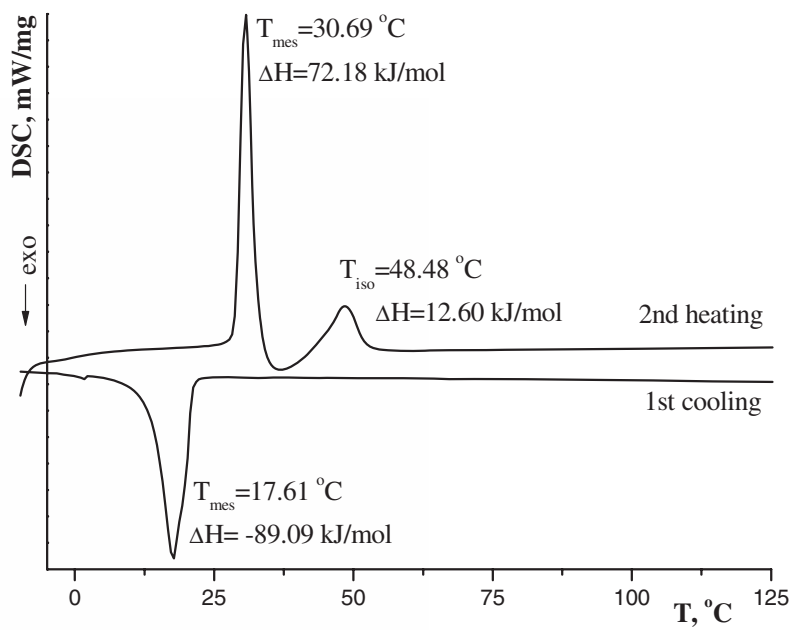

(c)

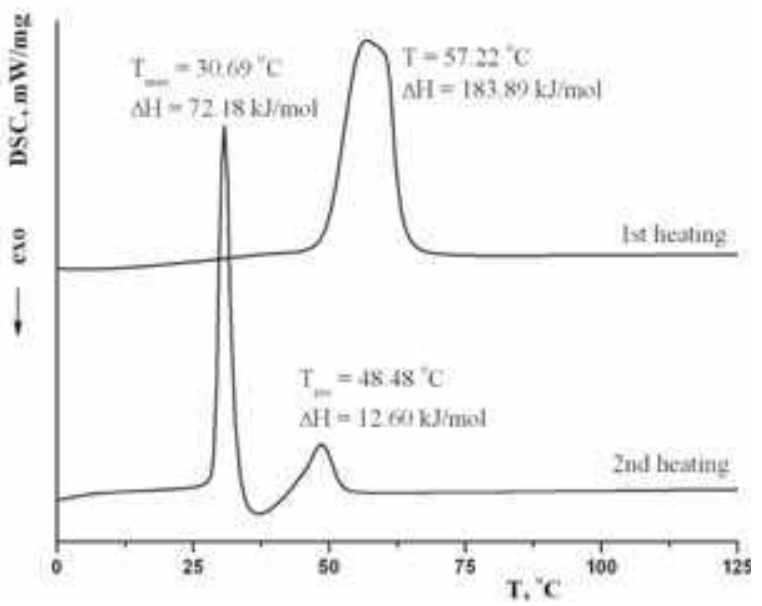

Figure 2. (a) Nongeometric texture of compound (3), heating cycle, $\mathrm{T}=47^{\circ} \mathrm{C}$; (b) DSC curves of compound (3) in heating and cooling cycle. (c) DSC curves of compound $(3)$ at $1^{\text {st }}$ and $2^{\text {nd }}$ heating.

Introduction of a phenyl ring with two polar groups into compound (7) yields compound $(\mathbf{8})$, which vitrifies from the mesophase. Compound $(\mathbf{8})$ melts at $\mathrm{T}=44^{\circ} \mathrm{C}$ and turns into an isotropic liquid at $\mathrm{T}_{\text {iso }}=50^{\circ} \mathrm{C}$. Upon cooling, a mesophase is formed at $\mathrm{T}_{\text {mes }}=36^{\circ} \mathrm{C}$ with a non-geometric texture (figure 5). Subsequent cooling leads to the appearance of a coloured anisotropic pattern which does not change under shear stress, which characterizes anisotropic glass $\left(\mathrm{Tg}=-10.41^{\circ} \mathrm{C}, \Delta \mathrm{Cp}\right.$ $=0.45 \mathrm{~J} /(\mathrm{g} * \mathrm{~K}))$. (a)

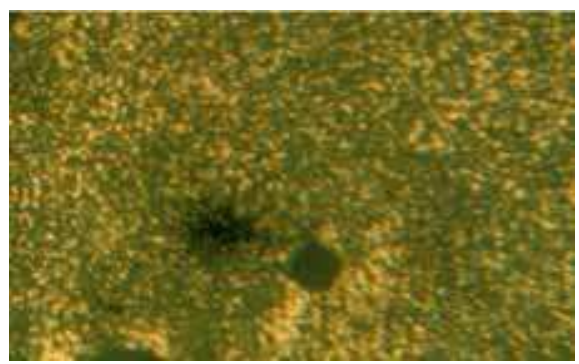

(b)

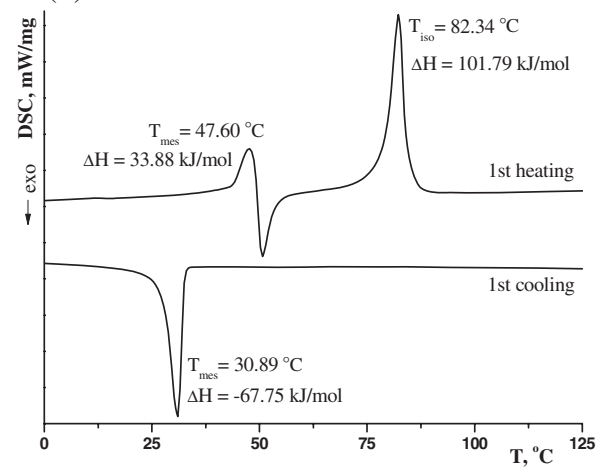

Figure 3. (a) Fine domain texture of compound (4), cooling cycle, $\mathrm{T}=29^{\circ} \mathrm{C}$; (b) DSC curves of compound (4) in heating and cooling cycles. 
(a)

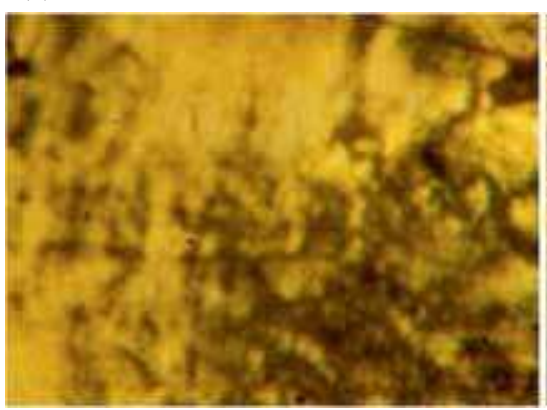

(b)

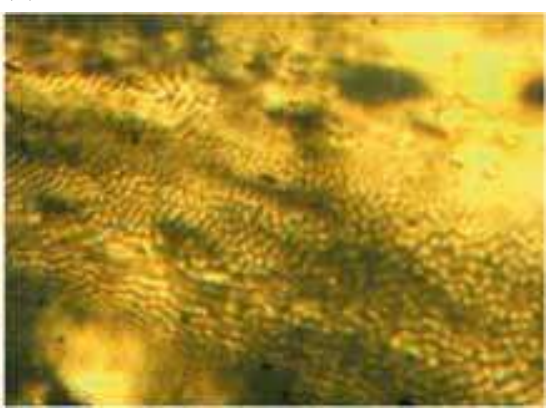

(c)

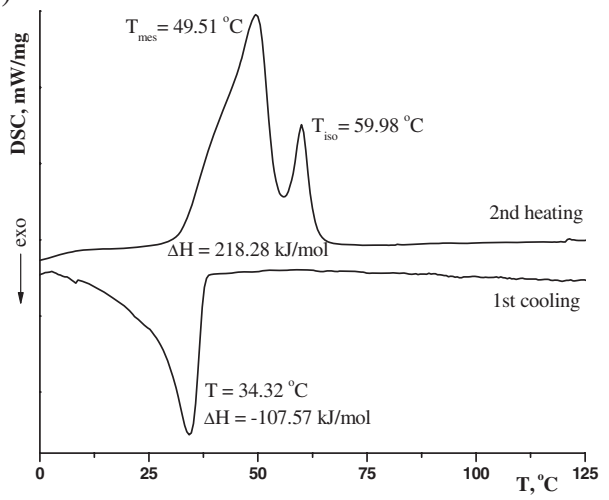

Figure 4. (a) Nongeometric texture of the thick sample (6) in cooling cycle, $\mathrm{T}=49^{\circ} \mathrm{C}$; (b) oily striations texture of the thin sample $(\mathbf{6}), \mathrm{T}=20^{\circ} \mathrm{C}$, cooling cycle; (c) DSC curves of the sample in heating and cooling cycles.

In conclusion, we have synthesized a new series of compounds derived from the esters of 3,4,5-tris(tetradecyloxy)benzoic acid (2)-(8). It was found that starting from ester (3), mesomorphic properties are revealed, and they depend on the presence of polar terminal groups in compounds (2), (4), (5), (7) and (8) which take part in the formation of hydrogen bonds. For compounds $(\mathbf{3})$ and (6), a mesophase is formed primarily by microsegregation. Increasing the molecular mass in series from (2) to (8) leads to anisotropic vitrification of compound (8).

The IR measurement of all compounds demonstrates the formation of complementary hydrogen bonds between fragments of polar groups in the case of carboxylic acid, aldehydes and esters. The IR absorption of

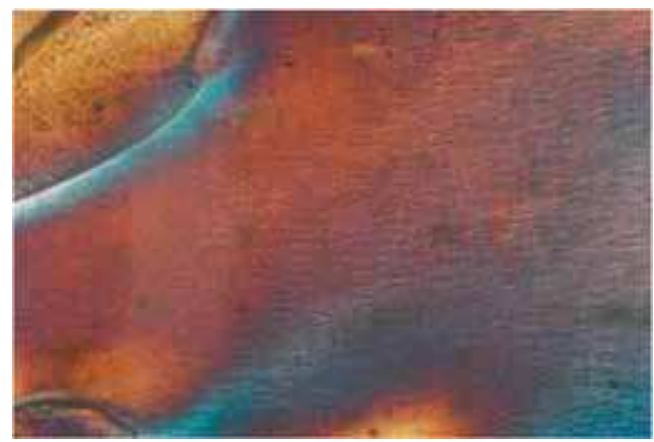

Figure 5. Texture of mesophase, cooling cycle, $\mathrm{T}=36^{\circ} \mathrm{C}$. acid (1) at $1688 \mathrm{~cm}^{-1}$ assigned to the $\mathrm{C}=\mathrm{O}$ stretching of the carboxylic acid shifted to $1751 \mathrm{~cm}^{-1}$ (4) and $1738 \mathrm{~cm}^{-1}$ (7), (figure 6), indicating that the carboxylic acids (1), (4) and (7) are quantitatively formed the hydrogen bond by formation of symmetrical and asymmetrical dimers at lengthening of molecules.

This situation is further supported by the fact that the $\mathrm{H}$-stretching peaks in the IR spectrum appears in the

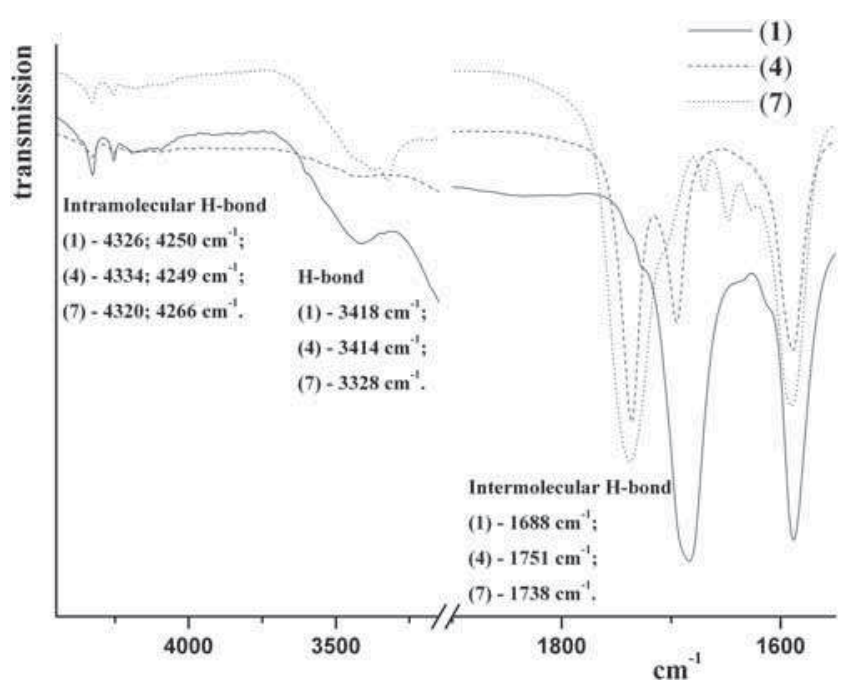

Figure 6. Fragments of FT-IR spectra of acids (1), (4) and (7) in the H-bonded region. 
H-bonded region (below $3400 \mathrm{~cm}^{-1}$ ). ${ }^{28}$ In particular, compound (1) $-(\mathbf{8})$ have been found to interact in dimers by double intermolecular $\mathrm{H}$-bonds and by intramolecular H-bonds. ${ }^{29}$

\section{Conclusions}

A new series of compounds derived from esters of 3,4,5-tris(tetradecyloxy)benzoic acid were synthesized. It was established that liquid-crystalline properties of this series of dendrons arise from of the degree of branching. This behavior can be explained by the formation of hydrogen bonds, as well as processes of microsegregation of the links of the macromolecule.

\section{Supplementary information (SI)}

All additional information pertaining to characterization of the compounds using NMR spectra, FT-IR spectra and mass-spectra are given in the supporting information. The electronic supporting information can be seen in www.ias.ac.in/chemsci.

\section{Acknowledgements}

This work was supported by the grant of Russian Foundation for Basic Research No. 14-03-31280-mol_a and the grant of the President of the Russian Federation (No. MK-70.2014.3). Dr. Akopova O.B. is thankful for the grant of Ministry of education and science RF (No. 4.106.2014K). EA, NMR, FT-IR spectroscopy and DSC analysis were carried out by the equipments of Interlaboratory scientific center «Verhnevolzhskij region center of physicochemical researching». The authors would like to thank Dr. Simon Dalgleish (Nagoya University) for useful discussion.

\section{References}

1. Newkome G R, Moorefield C N and Voegtle F 2001 In Dendrimers and dendrons: Concepts, syntheses and applications (Weinheim: Wiley-VCH) p. 636

2. Smith D K, Hirst A R, Love C S, Hardy J G, Brignell S V and Huang B 2005 Prog. Polym. Sci. 30220

3. Bauer S, Fisher H and Ringsdorf H 1993 Angew. Chem.Int. Ed. 321589

4. Pesak D J and Moore J S 1997 Angew. Chem. Int. Ed. 36 1636
5. Meier H and Lehmann M 1998 Angew. Chem. Int. Ed. 37643

6. Cameron J H, Facher A, Lattermann G and Diele S 1997 Adv. Mater. 9398

7. Tian Y Q, Kamata K, Yoshita H and Iyoda T 2006 Chem. Eur. J. 12584

8. Frauenrath H 2005 Prog. Polym. Sci. 30325

9. Turrin C-O, Maraval V, Leclaire J, Dantras E, Lacabanne C, Caminade A-M and Majoral J-P 2003 Tetrahedron $\mathbf{5 9} 3965$

10. Ropponen J, Tamminen J, Lahtinen M, Linnanto J, Rissanen K and Kolehmainen E 2005 Eur. J. Org. Chem. 200573

11. Ropponen J, Tuuttila T, Lahtinen M, Nummelin S and Rissanen K 2004 J. Polym. Sci. Part A. 42 5574

12. Percec V, Cho W-D, Ungar G and Yeardley D J P 2001 J. Am. Chem. Soc. 1231302

13. Percec V, Won B C, Peterca M and Heiney P A $2007 \mathrm{~J}$. Am. Chem. Soc. 12911265

14. Percec V, Peterca M, Dulcey A E, Imam M R, Hudson S D, Nummelin S, Adelman P and Heiney P A $2008 \mathrm{~J}$. Am. Chem. Soc. 13013079

15. Grebenkin M F and Ivaschenko A V 1989 In Liquid crystalline materials (Moscow: Khimiya) p. 228

16. Tschierske C 2001 J. Mater. Chem. 112647

17. Pegenau A, Hegmann T, Tschierske C and Diele S 1999 Chem. Eur. J. 51643

18. Chervonova U V, Gruzdev M S, Kolker A M, Manin N G and Domracheva N E 2010 Rus. J. Gen. Chem. 80 1954

19. Chervonova U V, Gruzdev M S and Kolker A M 2011 Rus. J. Gen. Chem. 811853

20. Vorobeva V E, Domracheva N E, Pyataev A V, Gruzdev M S and Chervonova U V 2015 Low Tem. Phys. 4115

21. Chervonova U V, Gruzdev M S and Kolker A M 2011 Rus. J. Gen. Chem. $\mathbf{8 1} 2288$

22. Schaz A, Valaityte E and Lattermann G 2005 Liq. Cryst. 32513

23. Tully D C, Wilder K, Fréchet J M J, Trimble A R and Quate C F 1999 Adv. Mater. 11314

24. Saudan C, Balzani V, Gorka M, Lee S-K, Maestri M, Vicinelli V and Vögtle F 2003 J. Am. Chem. Soc. 125 4424

25. Denieul M P, Laursen B, Hazell R and Skrydstrup T 2000 J. Org. Chem. 656052

26. Shreenivasa Murthy H N and Sadashiva B K 2004 Liq. Cryst. 311347

27. Seeboth Arno, Lotzsch Detlef, Ruhmann Ralf and Muehling Olaf 2014 Chem. Rev. 1143037

28. Ishihara S, Furuki Y and Takeoka S 2008 Polym. Adv. Technol. 191097

29. Beltrán E., Cavero E., Barberá J, Serrano J L, Elduque A and Giménez R 2009 Chem. Eur. J. 15 9017 